\title{
Functions of the C2H2 Transcription Factor Gene thmeal in Trichoderma harzianum under Copper Stress Based on Transcriptome Analysis
}

\author{
Jie Mei, Lirong Wang, Xiliang Jiang $(\mathbb{D}$, Beilei Wu, and Mei Li $\mathbb{D}$ \\ Institute of Plant Protection, Chinese Academy of Agricultural Sciences, Beijing 100081, China \\ Correspondence should be addressed to Xiliang Jiang; jiangxiliang@caas.cn and Mei Li; limei@caas.cn
}

Received 20 April 2018; Accepted 28 June 2018; Published 17 July 2018

Academic Editor: Nevijo Zdolec

Copyright (C) 2018 Jie Mei et al. This is an open access article distributed under the Creative Commons Attribution License, which permits unrestricted use, distribution, and reproduction in any medium, provided the original work is properly cited.

\begin{abstract}
Trichoderma spp. are important biocontrol filamentous fungi and have tremendous potential in soil bioremediation. In our previous studies, a $\mathrm{C} 2 \mathrm{H} 2$ type transcription factor coding gene (thmeal) was cloned from a biocontrol agent T. harzianum Th-33; the encoded sequence of thmeal contained 3 conserved C2H2 domains with Swi5 and Ace2 in Saccharomyces cerevisiae. The thmeal knockout mutant $\Delta$ thmeal showed $12.9 \%$ higher copper tolerance than the wild-type Th33. To elucidate the function of thmeal and its relationship with copper stress response, we conducted transcriptome sequencing and analysis of wild-type Th33 and $\Delta$ thmeal under $0.8 \mathrm{mM}$ copper stress. A total of 1061 differentially expressed genes (DEGs) were identified between the two strains, all DEGs were assigned to KEGG pathway database, 383 DEGs were annotated in 191 individual pathways, and the categories of ribosomal protein synthesis and amino acid metabolism were the most highly enriched ones. Analysis of related DEGs showed that the expression levels of intracellular glutathione detoxification enzyme, heat shock proteins, and ribosomal proteins in $\Delta$ thmeal were higher than that of the wild-type Th33, and the expression of metallothionein (MT) gene did not change. In addition, the expression levels of genes coding for proteins associated with the Ccc2p-mediated copper chaperone Atxlp transport of copper ions into the Golgi secretory pathway increased, as well as the copper amine oxidase (CuAO). These findings suggest that Thmeal is a negative regulated factor of copper tolerance ability in T. harzianum. It does not show metallothionein expression activator activities as that of Ace 2 in S. cerevisiae. We hypothesize that after T. harzianum has lost its thmeal gene, the ability of cells to scavenge reactive oxygen species, mainly through the glutathione antioxidant system, is enhanced, whereas protein synthesis and repair and copper secretion increase under copper stress, which increases the ability of the mutant strain to tolerate copper stress.
\end{abstract}

\section{Introduction}

Trichoderma spp. are important biocontrol filamentous fungi which are widely used to prevent soil-borne diseases in plants as active ingredients in biofertilizers and biopesticides. In addition, Trichoderma spp. also have tremendous potential in soil bioremediation [1-3]. In recent years, copper and copper-containing compounds have been extensively utilized in the preparation of microbicides and chemical pesticides and as livestock food additives, consequently resulting in a continuous increase of soil copper levels $[4,5]$. Researches on copper stress responses and metabolism mechanisms in Trichoderma species have facilitated the improvement of copper tolerance as well as the prevention and treatment of plant diseases.
Transcription factors (TFs), also known as trans-acting factors, are proteins that can directly or indirectly interact with cis-acting elements in gene promoters to regulate the start of gene transcription [6]. The thmeal gene of $T$. harzianum Th33 was cloned and was $1,446 \mathrm{bp}$ in length. It did not contain any intron and the encoded sequence contained three conserved $\mathrm{C} 2 \mathrm{H} 2$ zinc finger domains that were identical to the yeast activator of $\mathrm{HO}$ gene transcription Swi5 and metallothionein expression activator Ace2p. thmeal was speculated to be a $\mathrm{C} 2 \mathrm{H} 2$-type transcription factor gene (GenBank Accession Number MF802279). A knockout mutant of this gene, $\Delta$ thmeal, was obtained using a homologous double-crossover method. Studies have shown that when $\Delta$ thmeal was growing on potato dextrose agar (PDA) culture media containing $0-2.4 \mathrm{mM}$ copper ions, its 
growth rate significantly increased compared to the wildtype strain Th33, and its median inhibitory concentration $\left(\mathrm{MIC}_{50}\right)$ to copper ions was $1.92 \mathrm{mM}$, which was $12.9 \%$ higher than that of the wild-type strain. These findings showed that thmeal was associated with copper tolerance in Trichoderma and might participate in copper stress responses and copper metabolism. To further study the function of this gene, we conducted transcriptome sequencing of wild-type Th33 and $\Delta$ thmeal under $0.8 \mathrm{mM}$ copper treatment, analyzed DEGs, and examined the function of the thmeal gene in this study. The findings of the present study provide a foundation for the elucidation of copper stress responses and metabolism mechanisms in Trichoderma species.

\section{Materials and Methods}

2.1. Test Strains. The wild-type T. harzianum Th-33 was isolated from soil samples in the Beijing region as described previously [7]. The thmeal knockout mutant $\Delta$ thmeal was created with hygromycin B resistance by homologous recombination and then purified by isolation of single conidia and was stored in our laboratory.

2.2. Determination of Trichoderma Growth Rate. T. harzianum Th33 wild-type strain and the mutant $\Delta$ thmeal were inoculated on the PDA media for activation for three days at $28^{\circ} \mathrm{C}$. Then, a culture disc was removed with a cork borer (5-mm diameter) and inoculated on the center of PDA plates with copper iron concentrations of $0 \mathrm{mM}, 0.8 \mathrm{mM}$, 1.6 mM, 2.4 mM, 3.2 mM, and 4.0 mM. Quadruplicates were prepared for each concentration, and the plates were grown at $28^{\circ} \mathrm{C}$. The growth status of the fungi was observed at 24-h intervals, and the diameter of the fungal colonies was recorded. The $\mathrm{MIC}_{50}$ of $\mathrm{T}$. harzianum to copper ions was calculated as described elsewhere [8]. A standard curve of the colony growth inhibition rate to its corresponding copper concentration was constructed, and inhibition rate was calculated using the following equation: Inhibition rate $(\%)=[($ Diameter of control colony $(\mathrm{cm})-$ Diameter of treated colony $(\mathrm{cm})] /$ Diameter of control colony $(\mathrm{cm}) \times$ $100 \%$. The copper concentration at a growth inhibition rate of $50 \%$ was calculated using a regression formula, which is the $\mathrm{MIC}_{50}$.

2.3. Strain Treatment and Sampling. Approximately $100 \mu \mathrm{L}$ of Th33 and $\Delta$ thmeal spore suspensions at a density of $1 \times$ $10^{7} / \mathrm{mL}$ were inoculated into $250-\mathrm{mL}$ conical flasks containing $100 \mathrm{~mL}$ potato dextrose (PD) liquid culture media. Each strain was inoculated in duplicate. The flasks were cultured in a shaking incubator at $28^{\circ} \mathrm{C}$ and $180 \mathrm{rpm}$ for $39 \mathrm{~h}$. This was followed by the addition of $1 \mathrm{~mL}$ of filter-sterilized copper sulfate solution $(80 \mathrm{mM})$ to a final copper concentration of $0.8 \mathrm{mM}$. The flasks were then cultured for another $4 \mathrm{~h}$. The above fermentation broth samples were filtered and washed thrice to collect the hyphae. Aliquots of the samples were then prepared, flash frozen in liquid nitrogen, and then stored at $-80^{\circ} \mathrm{C}$.
2.4. Total RNA Extraction. Total RNA was extracted using Trizol reagent (Invitrogen, CA, USA) according to the manufacturer's instruction. The RNA quality and quantity were determined using an Agilent 2100 Bioanalyzer. The Qubit RNA Assay Kit was used for accurate quantification of the initial total RNA.

2.5. Construction of cDNA Libraries and Sequencing. We commissioned Berry Genomics Co., Ltd., to conduct transcriptome sequencing using the Illumina HiSeq 2500 platform. Dynabeads mRNA DIRECT kits (Invitrogen, CA, USA) were used to extract mRNA from total RNA. After mRNA fragmentation, random primers were used to synthesize the first-strand cDNA through reverse transcription before synthesis of the second-strand cDNA to obtain double-stranded cDNA. End-repair and addition of adenines to the $3^{\prime}$ terminus of the double-stranded cDNA was performed, followed by ligation of sequencing adapters. After purification of the ligated products, PCR amplification was conducted, and the PCR products were separated using 2\% agarose gel electrophoresis. The target $400-500$ bp band was extracted from the gel and recovered as the final library. qPCR was used for quality control of the libraries prior to loading on the machine for sequencing.

2.6. Gene Annotation and Quantitative Analysis of Expression Levels. Tophat software was used for alignment and annotation of the reads from the sequenced samples and T. harzianum Th33 genome data (GenBank Acc. No. PRJNA272949) [7]. Gene expression levels were expressed as fragments per kilobase of transcript per million fragments mapped (FPKM) [9], i.e., the number of matches for every kilobase of transcript per million fragments.

2.7. Analysis of Differentially Expressed Genes (DEGs). DEGs between samples were generated using Cuffdiff, in which the fold-change was the ratio of the expression levels between the two samples; i.e., $\log _{2}$ (FC) was $\log _{2}$ (fold-change) = $\log _{2}$ (Sample A/Sample B). Here, we set $p$ value $\leq 0.01$ and $\left|\log _{2}(\mathrm{FC})\right| \geq 1$ as significant differences. The DEGs were identified and functionally annotated using the databases of Gene Ontology (GO) and KEGG.

2.8. Validation of Transcriptome Sequencing Results. To confirm the reliability of transcriptome sequencing results, we selected 12 DEGs for real-time fluorescence quantitative PCR (qRT-PCR) validation. qRT-PCR was conducted using the SYBR Green reagent kit (SuperReal PreMix Plus SYBR Green kit FP205, Tiangen). Each 20- $\mu$ L PCR reaction system consisted of $10 \mu \mathrm{L} 2 \times$ SuperReal PreMix Plus, $1 \mu \mathrm{L}$ of each forward and reverse primer (10 pM each), $1 \mu \mathrm{L}$ of the template cDNA ( $80 \mathrm{ng}$ ), and $7 \mu \mathrm{L}$ of double-distilled water. The reaction was performed on a ABI7500 PCR system, and the reaction conditions were as follows: predenaturation at $95^{\circ} \mathrm{C}$ for $15 \mathrm{~min}$, followed by 40 cycles of denaturation at $95^{\circ} \mathrm{C}$ for $10 \mathrm{~s}$, and annealing and extension at $60^{\circ} \mathrm{C}$ for 32 s. Technical triplicates were prepared for each sample. The gene expression levels of the wild-type Th33 subjected to 


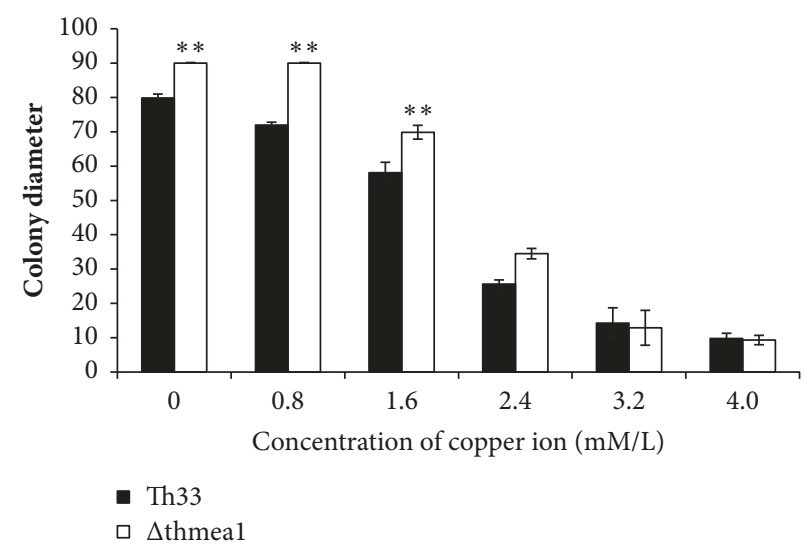

(a)

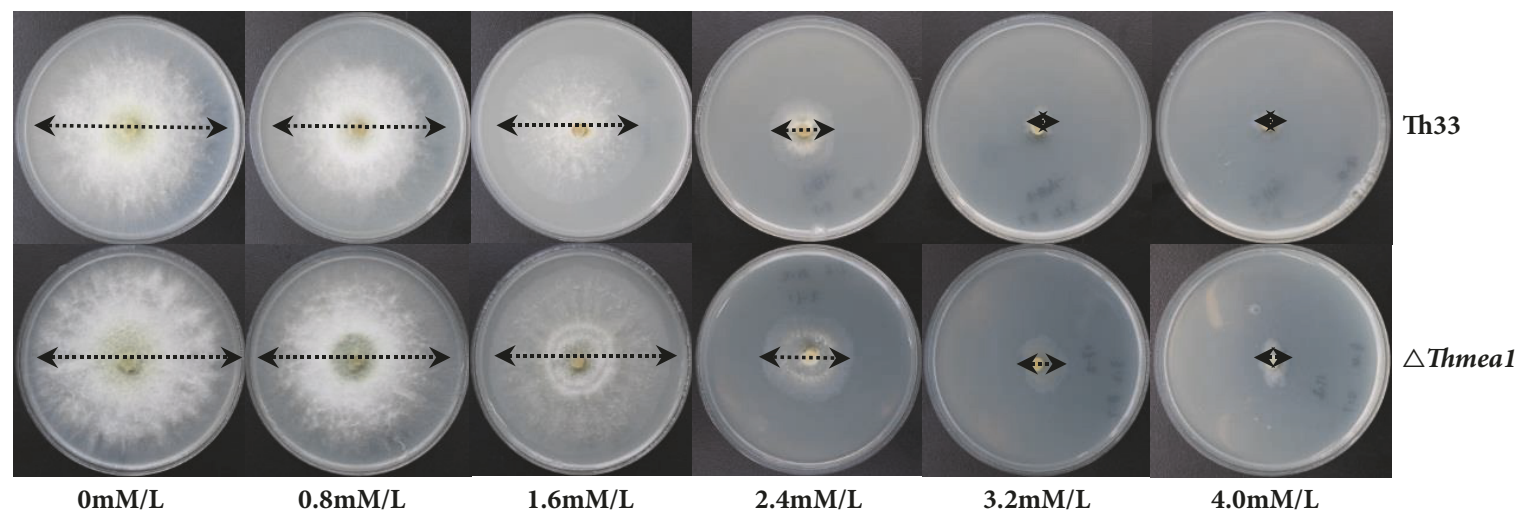

(b)

FIGURE 1: Effects of copper ions on the growth rate and colony morphology of Trichoderma harzianum Th33 and thmeal gene knockout mutant $\Delta$ thmeal. (a) Colony diameter of $T$. harzianum Th33 wild-type and mutant $\Delta$ thmeal under different copper iron concentrations at $28^{\circ} \mathrm{C}$ for 2 days. (b) Colony morphology of T. harzianum Th33 wild-type and mutant $\Delta$ thmeal strains under different copper concentrations at $28^{\circ} \mathrm{C}$ for 2 days. Note: figure data is expressed as mean \pm standard error. * represents significance level of differences under different treatments $(*$ $\mathrm{p}<0.05 ; * * \mathrm{p}<0.01)$.

copper treatment were used as a reference, and the $2^{-\triangle \triangle \mathrm{CT}}$ method was used for the calculation of gene expression levels in other samples. The internal reference gene was the T. harzianum glyceraldehyde 3-phosphate dehydrogenase (GAPDH) gene. Primers used in this study are shown in Table $\mathrm{S} 1$ in Supplementary Materials.

\section{Results and Analysis}

3.1. Comparison of Growth Rates between the T. harzianum Th33 Wild-Type Strain and the Mutant Strain $\Delta$ thmeal. The growth rate of the mutant $\Delta$ thmeal in PDA culture media was higher than the wild-type strain, and copper ions imparted inhibitory effects on the growth of both strains. The colony diameters of the wild-type Th33 and the mutant $\Delta$ thmeal strains grown on PDA plates with copper concentrations of $0 \mathrm{mM}, 0.8 \mathrm{mM}, 1.6 \mathrm{mM}, 2.4 \mathrm{mM}, 3.2 \mathrm{mM}$, and $4.0 \mathrm{mM}$ after culturing for 2 days at $28^{\circ} \mathrm{C}$ were showed in Figure 1(a). With increasing copper concentrations, the growth rate of the colonies decreased. Within the copper concentration range of 0-2.4 mM, the growth rate of $\Delta$ thmeal was significantly faster than wild-type Th33. However, at copper iron concentrations of $3.2 \mathrm{mM}$ and $4.0 \mathrm{mM}$, the growth of both strains was severely inhibited, without significant differences. Under low copper concentrations, $\Delta$ thmeal exhibited a higher colony growth rate and greater amount of aerial hypha compared to the wild-type strain after 2 days of growth, as shown in Figure 1(b). The formula for calculating the growth inhibition rate of the wild-type Th33 was $\mathrm{y}=0.2755 \mathrm{x}+0.0316, \mathrm{r}=$ 0.969 , in which y was the growth inhibition rate of the colony and $\mathrm{x}$ was the copper concentration. The regression formula for growth inhibition rate of $\Delta$ thmeal was $\mathrm{y}=0.282 \mathrm{x}-$ $0.0409, \mathrm{r}=0.976$. The $\mathrm{MIC}_{50}$ of the wild-type Th33 strain and $\Delta$ thmeal was $1.70 \mathrm{mM}$ and $1.92 \mathrm{mM}$, respectively. The copper tolerance of $\Delta$ thmeal showed a $12.9 \%$ increase compared to Th33, showing that $\Delta$ thmeal had a significantly higher copper tolerance than the wild-type strain.

\subsection{Total RNA Extraction and Construction of Sequencing} Libraries. The samples used for transcriptome sequencing were hyphae from wild-type Th33 that was grown in liquid culture containing $0.8 \mathrm{mM}$ copper irons (Th33-0.8-1 and Th33-0.8-2) and hyphae from $\Delta$ thmeal that was grown in liquid culture containing $0.8 \mathrm{mM}$ copper irons $(\Delta T H-0.8-1$ 
and $\Delta T H-0.8-2)$. Two biological replicates were prepared for one strain, generating a total of four samples. The electropherogram of the total RNA of the 4 samples showed clear separate bands of $18 \mathrm{~S}$ and $28 \mathrm{~S}$ rRNA, whereas that of the $5 \mathrm{~S}$ band was weaker, indicating low RNA degradation and thus the RNAs were intact (figure not shown). The RIN values of the samples were $\geq 9.8$ and quality evaluation was Grade I. Therefore, these qualified RNA samples were used for library construction.

3.3. Quality Evaluation of the Raw Data. Transcriptome sequencing was conducted on an Illumina HiSeq 2500 platform. The total yields of the four samples were $2,695,311,250 \mathrm{bp}, \quad 2,803,014,250 \mathrm{bp}, \quad 3,137,172,000 \mathrm{bp}$, and $2,952,492,750 \mathrm{bp}$, respectively, and the base contents were all $>2.6 \mathrm{~Gb}$. For base quality scores, bases with a Phred score of Q20 (\%) were all $\geq 92 \%$. Therefore, the sequencing quality of this experiment was satisfactory and thus can be used for subsequent quantitative analysis of expression levels.

3.4. Gene Annotation and Quantitative Analysis of Expression Levels. The T. harzianum Th33 genome sequence was used as reference, and Tophat 2.0.12 software was used for alignment of sequencing reads. The proportion of reads from various samples that were aligned and mapped to the genome was $>80 \%$ (Table S2). Quantitation of gene expression was conducted using the FPKM method. The results of analysis of 10,849 genes showed that the proportion of genes that had a gene expression abundance of $10-50$ was the highest, accounting for $31 \%$ of the total number of genes (Table S3).

3.5. Analysis of DEGs. Cufflinks were used for analysis of DEGs between samples $(P \leq 0.01)$. Under copper stress, a total of 1,061 DEGs were identified between the knockout mutant $\Delta$ thmeal and wild-type $T h 33$, of which 526 genes were upregulated and 535 genes were downregulated. Statistical analysis indicated that for differential expression genes with a one- to twofold absolute value of $\log _{2}(\mathrm{FC})$, there were 268 upregulated genes and 355 downregulated genes; for genes with a two- to threefold $\log _{2}(\mathrm{FC})$ value, there were 73 upregulated genes and 87 downregulated genes; for genes with a more than threefold $\log _{2}(\mathrm{FC})$ value, there were 34 upregulated genes and 14 downregulated genes. The results showed that DEGs with a one-to twofold $\log _{2}(\mathrm{FC})$ accounted for a higher proportion, as shown in Table S4 and Figure 2.

3.6. GO Enrichment Analysis of DEGs. Blast2GO [7, 10] program was used for GO analysis. The program extracted the GO terms associated with the homologies identified by BLAST and returned a list of GO annotations, which were presented as hierarchical categories of increasing specificity. GO enrichment analyses were performed using Fisher's exact test with multiple testing corrections and an FDR of 0.05. A total of 666 DEGs were categorized into 31 functional groups in three main categories, "cellular component," "molecular function," and "biological process" (Table S5 and Figure 3). Some unigenes were assigned to multiple categories of GO terms, whereas others could not be assigned to a given GO term. In the cell component category, "cell" (113, 16.97\%),

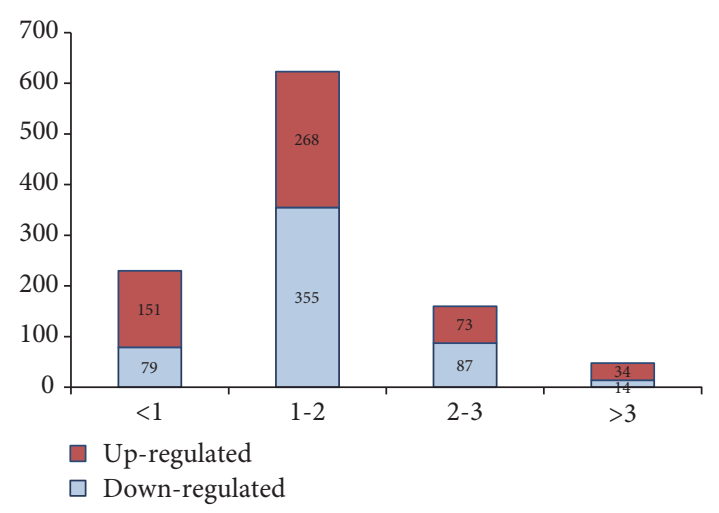

Figure 2: Differentially expressed genes of $\Delta$ TH-0.8_VS_Th33-0.8.

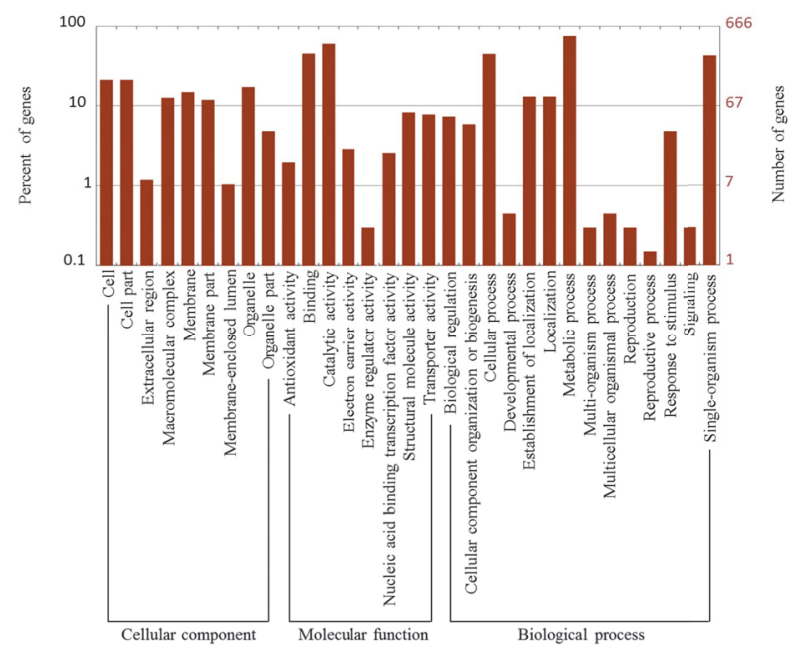

FIGURE 3: GO enrichment analysis of differentially expressed genes.

"cellular components" (113, 16.97\%), and "organelle" (92, $13.81 \%)$ were the most abundant terms. In the molecular function category, genes associated with "binding" (145, 21.77\%) and "catalysis" (246, 36.94\%) were the most abundant terms. In the biological process category, "cellular processes" (250, $37.54 \%)$, "metabolic processes" (451, 67.72\%), and "singleorganism process" $(233,34.98 \%)$ were the most abundant terms, as shown in Table S5 and Figure 3.

3.7. KEGG Pathway Enrichment Analysis of DEGs. KEGG pathway analysis annotated 338 DEGs to 191 metabolic pathways. Table S6 and Figure 4 showed the top 17 metabolic pathways that showed relatively high enrichment significance $(\mathrm{P} \leq 0.1)$. Among these pathways, ribosomal protein synthesis-associated pathways and genes accounted for $18.02 \%$ of the DEGs, genes involved in amino acid metabolism accounted for $20.62 \%$ (tryptophan metabolism; phenylalanine metabolism; glycine, serine and threonine metabolism, and tyrosine metabolism), and genes associated with cell cycle accounted for $7.31 \%$ (cell cycle, and cell cycleyeast). 


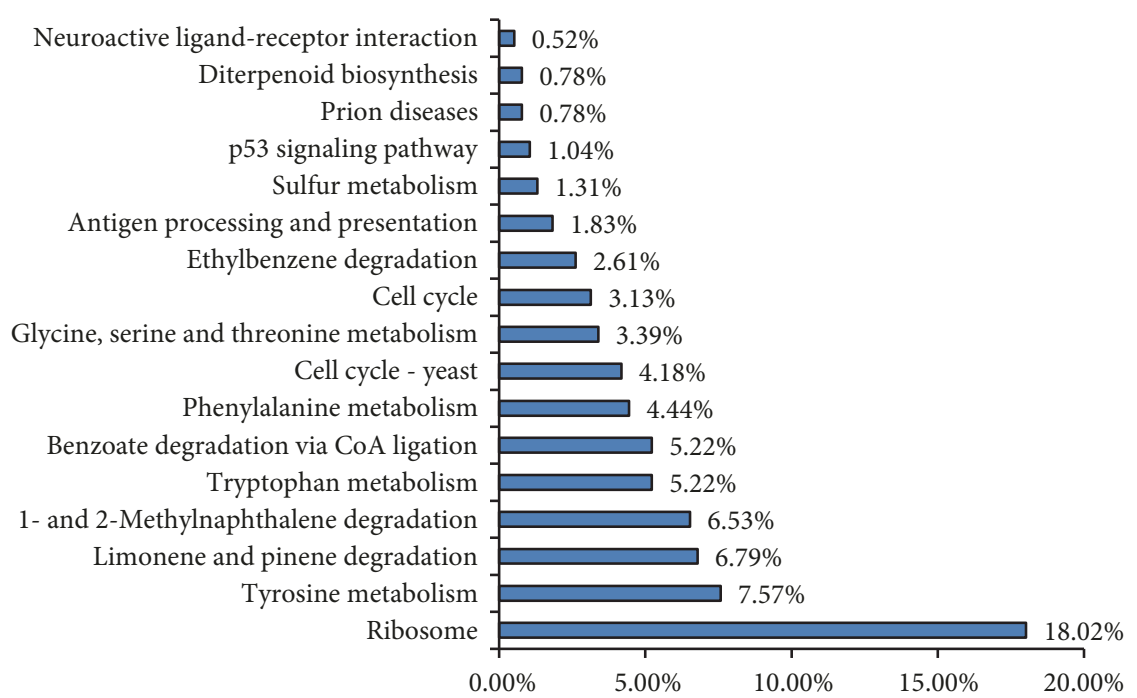

FIGURE 4: Pathway enrichment analysis of DEGs. The percentage of differentially expressed genes involved in KEGG pathways. Only the top 17 most abundant KEGG pathways are represented.

3.8. Genes Associated with Antioxidant Enzymes. The expression of antioxidant enzymes in the mutant $\Delta$ thmeal and wildtype Th33 strains including catalase (Tha_07751, CAT), five peroxidases (Tha_04316, Tha_00485, Tha_00674, Tha_02847, and Tha_10717, POD), and copper and zinc superoxide dismutase genes (Tha_04932, SOD) was analyzed. Among these genes, Tha_07751, Tha_10717, and Tha_02847 were downregulated, whereas Tha_04316 was upregulated. This showed that when $\Delta$ thmeal encountered copper stress, the expression levels of some antioxidant enzymes underwent varying degrees of up- or downregulation. In addition, analysis of genes associated with glutathione (GSH) showed that there were three out of four glutathione S-transferase (GST) genes in the mutant $\Delta$ thmeal strain (Tha_03629, Tha_05984, and Tha_10746) and glutathione peroxidase (Tha_06359) that were upregulated, whereas one GST gene (Tha_01711) was downregulated (Table 1). This showed that the overall expression levels of genes associated with GSH enzymes in $\Delta$ thmeal increased under copper stress.

3.9. Ribosomal Proteins and Heat Shock Protein- (HSP-) Related Genes. Ribosomal proteins are important components of ribosomes and play important roles in intracellular protein synthesis. HSPs have been reported to be generated under multiple stress conditions and participate in the repair and degradation of stress-damaged proteins as well as the folding, transportation, and assembly of newly synthesized peptide chains [11]. Under copper stress, there were 65 genes associated with ribosomal proteins in the mutant $\Delta$ thmeal that showed differential expression, of which 60 genes were upregulated. In addition, the FPKM values of these genes were around twofold higher than that in the wild-type fungi, which were around 1,100-4,300 (Table S6). Under copper stress, the expression levels of 12 HSPs in the mutant $\Delta$ thmeal were higher than the wild-type strain. Among these genes, Tha_00375, Tha_03314, Tha_09210, Tha_10420, and Tha_10740 showed significant expression (Table 2). These results suggested that the loss of the thmeal gene increased the synthesis capacity of ribosomal proteins and HSPs in $\Delta$ thmeal under copper stress, thereby ensuring protein synthesis, supplementation, and repair.

3.10. Genes Associated with Copper Metabolism. Copper metabolism mainly includes uptake, intracellular storage and utilization, and extracellular release of copper ions. The expression of copper metabolism associated genes under copper stress were analyzed and showed in Table 3. Under copper stress, the copper reductase gene Tha_07043 (Fre2) was significantly upregulated in the $\Delta$ thmeal mutant, but both strains showed relatively low expression levels. Among genes encoding for the four copper transporter proteins (Ctrp), Tha_02284 was significantly downregulated, but the FPKM values of the two strains were relatively low, whereas there were no significant differences in expression levels in Tha_01417, Tha_03591, and Tha_05828 between the two strains. This suggested that there may be no significant difference in the uptake of copper ions between the two strains. The genes for the copper chaperone proteins Tha_01680 (Ccs1) and Tha_07893 (Cox17) were not differentially expressed in the wild-type and $\Delta$ thmeal strains, whereas the genes for the copper chaperone protein Tha_00261 (Atxl) and the Ptype ATPase Tha_08191 (Ccc2) were upregulated in $\Delta$ thmeal. This indicated that there were more copper ions entering the Golgi vesicles in the mutant strain. In addition, in the two strains, the multicopper oxidase gene Tha_02340 (Fet3) that participates in utilization and secretion of copper ions in Golgi vesicles was expressed at low transcriptional level. However, the copper amine oxidase gene Tha_03788 showed upregulated expression in the knockout mutant $\Delta$ thmeal. These findings suggested that Tha_03788 may have a similar function to the multicopper oxidase gene Fet3. The aforementioned results showed that, under copper stress, the knockout mutant $\Delta$ thmeal may exhibit stronger secretion and release of intracellular copper ions, thereby increasing its tolerance 
TABLE 1: Expression of genes associated with antioxidant enzymes under copper stress.

\begin{tabular}{lccccc}
\hline Gene ID & Th33-0.8 & $\begin{array}{c}\Delta \text { TH-0.8 } \\
\text { FPKM }\end{array}$ & $\log _{2}($ FC $)$ & P_value & Annotation \\
\hline Tha_07751 & 258.9260 & 99.9329 & -1.3735 & 0.00005 & Catalase \\
Tha_04316 & 62.6029 & 195.1620 & 1.6404 & 0.00005 & Peroxidase \\
Tha_00485 & 216.0380 & 431.4815 & 0.9980 & 0.00005 & Peroxidase \\
Tha_00674 & 293.2600 & 519.5290 & 0.8250 & 0.00005 & Peroxidase \\
Tha_02847 & 256.7990 & 124.8930 & -1.0399 & 0.00005 & Peroxidase \\
Tha_10717 & 165.2110 & 40.6521 & -2.0229 & 0.00005 & Peroxidase \\
Tha_04932 & 897.1265 & 477.6965 & -0.9092 & 0.00005 & Copper, zinc superoxide dismutase \\
Tha_03629 & 6.9580 & 38.1848 & 2.4563 & 0.00005 & Glutathione S- transferase \\
Tha_05984 & 35.1119 & 188.6655 & 2.4258 & 0.00005 & Glutathione S- transferase \\
Tha_10746 & 141.4985 & 613.1045 & 2.1154 & 0.00005 & Glutathione S- transferase \\
Tha_06359 & 211.2125 & 502.8465 & 1.2514 & 0.00005 & Glutathione peroxidase \\
Tha_01711 & 72.0724 & 31.9052 & -1.1757 & 0.00005 & Glutathione S- transferase \\
\hline
\end{tabular}

$\log _{2}(\mathrm{FC})$ values of $\mathrm{p}$ value $<0.01$ and $|\log 2(\mathrm{FC})| \geq 1.0$ were considered to be statistically significant.

TABLE 2: Expression of genes associated with heat shock proteins under copper stress.

\begin{tabular}{lccccc}
\hline Gene ID & $\begin{array}{c}\text { Th33-0.8 } \\
\text { FPKM }\end{array}$ & $\begin{array}{c}\Delta \text { TH-0.8 } \\
\text { FPKM }\end{array}$ & $\log _{2}(\mathrm{FC})$ & P_value & Annotation \\
\hline Tha_00375 & 3928.4350 & 17740.9000 & 2.1751 & 0.00005 & HSP 30 \\
Tha_01588 & 39.5399 & 72.1441 & 0.8676 & 0.0001 & Endoplasmic reticulum HSP \\
Tha_03314 & 1349.0900 & 4187.4700 & 1.6341 & 0.00005 & HSP 70 \\
Tha_04863 & 329.5615 & 572.9580 & 0.7979 & 0.00005 & HSP 101 \\
Tha_05072 & 99.0282 & 180.4830 & 0.8660 & 0.00005 & HSP 78 \\
Tha_09210 & 50.5491 & 148.5090 & 1.5548 & 0.00005 & Small HSP \\
Tha_10420 & 2218.2100 & 8353.4400 & 1.9130 & 0.00005 & HSP 80 \\
Tha_10740 & 446.6465 & 1410.3950 & 1.6589 & 0.00005 & hsp70 family protein, mitochondrial precursor \\
Tha_03207 & 83.88795 & 201.6835 & 1.26556 & 0.00005 & hsp60 mitochondrial precursor-like protein \\
Tha_07533 & 96.4268 & 186.609 & 0.95251 & 0.00005 & hsp70-like protein \\
Tha_01643 & 95.8451 & 46.79905 & -1.03422 & 0.00005 & hsp70 family protein \\
Tha_00917 & 414.4935 & 883.909 & 1.09255 & 0.00005 & \\
\hline
\end{tabular}

$\log _{2}(\mathrm{FC})$ values of $\mathrm{p}$ value $<0.01$ and $|\log 2(\mathrm{FC})| \geq 1.0$ were considered to be statistically significant.

TABLE 3: Expression of genes associated with copper metabolism under copper stress.

\begin{tabular}{|c|c|c|c|c|c|}
\hline Gene ID & $\begin{array}{c}\text { Th33-0.8 } \\
\text { FPKM }\end{array}$ & $\begin{array}{c}\text { TH-0.8 } \\
\text { FPKM }\end{array}$ & $\log _{2}(\mathrm{FC})$ & P_value & Annotation \\
\hline Tha_07043 & 0.5825 & 4.8977 & 3.0718 & 0.00005 & iron reductase Fre2 \\
\hline Tha_02284 & 18.6548 & 7.2156 & -1.3703 & 0.00075 & copper transporter protein Ctrp \\
\hline Tha_01417 & 102.2066 & 138.307 & 0.4364 & 0.12985 & copper transporter protein Ctrp \\
\hline Tha_03591 & 33.4524 & 40.7149 & 0.2834 & 0.2423 & copper transporter protein Ctrp \\
\hline Tha_05828 & 59.133 & 44.4831 & -0.4107 & 0.0701 & copper transporter protein Ctrp \\
\hline Tha_01680 & 54.4918 & 51.1831 & -0.09037 & 0.394517 & copper chaperone protein Ccs1 \\
\hline Tha_07893 & 83.8104 & 90.8476 & 0.1163 & 0.286482 & copper chaperone protein Cox17 \\
\hline Tha_00261 & 313.5735 & 687.164 & 1.1318 & 0.00005 & copper chaperone protein Atxl \\
\hline Tha_08191 & 113.641 & 400.6475 & 1.8178 & 0.00005 & P-type ATPases Ccc2 \\
\hline Tha_02340 & 0.3347 & 0.4288 & 0.3574 & 0.418922 & multi-copper oxidase gene Fet3 \\
\hline Tha_03788 & 55.4367 & 167.685 & 1.5968 & 0.00005 & copper amine oxidase $\mathrm{CuAO}$ \\
\hline Tha_07074 & 325.4160 & 332.0320 & -0.0290 & 0.91225 & Metallothionein MT \\
\hline
\end{tabular}

$\log _{2}(\mathrm{FC})$ values of $\mathrm{p}$ value $<0.01$ and $|\log 2(\mathrm{FC})| \geq 1.0$ were considered to be statistically significant. 


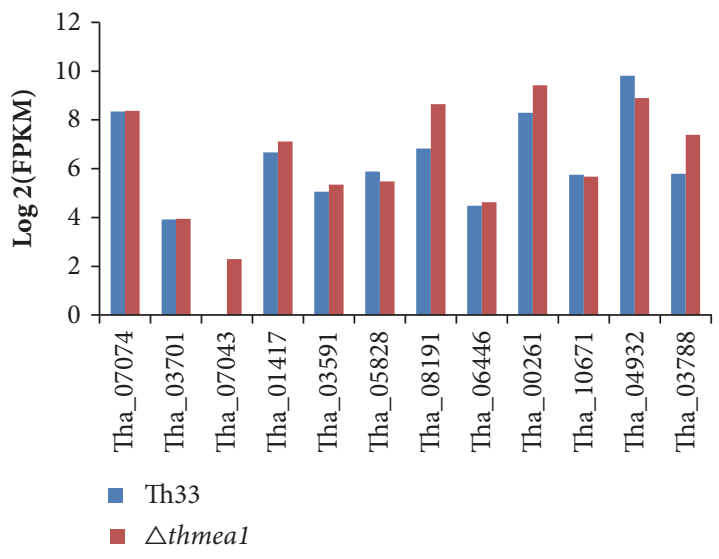

(a)

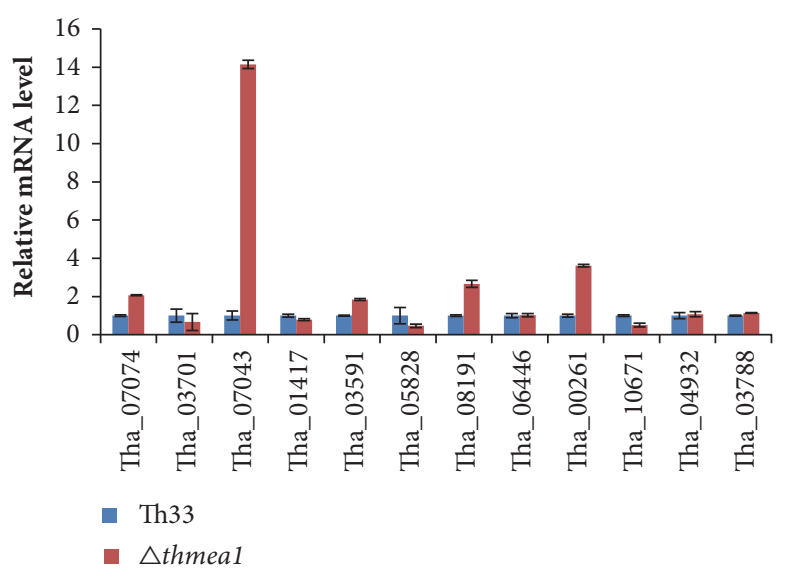

(b)

FIGURE 5: Validation of transcriptome sequencing results. (a) The transcriptome expression levels of 12 differentially expressed genes were expressed as $\log _{2}$ FPKM; (b) qRT-PCR analysis of 12 differentially expressed genes. Note: figure data is expressed as the mean \pm standard error.

to copper ions. The metallothionein gene Tha_07074 (MT) had higher expression levels in both strains but no significant differences in expression levels between wild Th33 and $\Delta$ thmeal, suggesting that this protein may play an important role in the release of copper ions in cells and was not regulated by Thmeal.

3.11. Validation of Transcriptome Sequencing Results. To validate the reliability of transcriptome sequencing results, we selected 12 DEGs for qRT-PCR analysis. These DEGs included the metallothionein gene (Tha_07074), macl transcription factor (Tha_03701), iron reductase (Tha_07043), the genes for three transporter proteins, (Tha_01417, Tha_03591, and Tha_05828), the genes for two P-type ATPases (Tha_08191, Tha_06446), the copper chaperone gene (Tha_00261), genes for two superoxide dismutase (Tha_10671, Tha_04932), and the copper amine oxidase gene (Tha_03788). The melting curves of each gene were analyzed by qRT-PCR, and a single peak was observed for each gene product (Figure S1). qRT-PCR analysis showed that the expression levels and trends of these DEGs coincide with transcriptome sequencing results $\left(\log _{2}\right.$ FPKM), as shown in Figure 5.

\section{Discussion}

To detect changes in copper tolerance in the T. harzianum Th33 thmeal mutant $\Delta$ thmeal, transcriptome sequencing of the T. harzianum Th33 wild-type and mutant $\Delta$ thmeal strains under copper stress was performed. The T. harzianum GAPDH coding gene gapdh was used as internal reference for qRT-PCR validation of 12 selected T. harzianum genes. The validation results coincided with the transcriptome sequencing results, thereby demonstrating the reliability of our findings. The expression levels of the antioxidant enzymes, glutathione-related enzymes, ribosomal proteins, HSPs, and copper metabolism associated genes in wild-type Th33 and $\Delta$ thmeal were analyzed to examine the function of the thmeal gene in this study.
Copper stress causes the accumulation of large amounts of reactive oxygen species in cells [11, 12]. Generally, it is believed that cells activate and increase the levels of antioxidant enzymes such as CAT, POD, and SOD and reduce that of glutathione (GSH) to alleviate oxidative stress [13]. GST is a key enzyme that catalyzes the binding of glutathione and metal ions and plays an important role during the binding of glutathione to copper ions [14]. In addition, glutathione peroxidase has functions such as reducing toxic peroxide compounds and protecting the structure and function of the cell membrane $[15,16]$. In this study, the expression of antioxidant enzymes in $\Delta$ thmeal showed varying degrees of up- and downregulation under copper stress, whereas GST showed a general upregulated expression. These findings suggested that $\Delta$ thmeal changed the activity of antioxidant enzymes and increases the expression levels of GST to increase the copper-binding capacity and ability to clear toxic peroxide compounds, thereby alleviating the damage caused by reactive oxygen species to cells.

Ribosomes are major sites in the cell where proteins are synthesized and ribosomal proteins are important components of ribosomes [17]. HSPs participate in the repair and degradation of stress-damaged proteins, as well as the folding, transportation, and assembly of newly synthesized peptide chains [18]. These two types of proteins play important roles during protein synthesis. Under copper stress, the expression of eight HSP genes and 60 ribosomal protein genes in the mutant $\Delta$ thmeal was upregulated. These findings suggested that the loss of the thmeal gene in T. harzianum resulted in an increase in protein synthesis and repair capabilities.

Bioinformatics analysis showed that the amino acids of Thmeal contained 3 conserved $\mathrm{C} 2 \mathrm{H} 2$ domains, which were identical to $\mathrm{C} 2 \mathrm{H} 2$ transcription factors Swi5 and Ace2 in Saccharomyces cerevisiae. Swi5 was an activator of $\mathrm{HO}$ gene transcription, and Ace2 was a metallothionein expression activator; Swi5 and Ace2 had functional similarities [19, 20]. Metallothioneins (MTs) are rich in cysteine and can effectively bind $\mathrm{Cu}^{+}$ions to decrease free copper ions and alleviate heavy metal stress [21]. This had been observed in 
the yeast strain with tandem expression of the yeast MT gene, Cup1, which could tolerate $12 \mathrm{mM}$ of copper ions, whereas normal strains could only tolerate $1.75 \mathrm{nM} / \mathrm{L}$ of copper ions [22]. In this study, the MT gene (Tha_07074) had higher expression levels in both wild Th33 and the $\Delta$ thmeal, but there were no significant differences between the two strains, indicating that MT protein played an important role in responding to copper stress and was not affected by thmeal in T. harzianum. Thmeal did not show the same function as Swi5 and Ace2.

Organisms have developed a set of strict and relatively conserved protective mechanisms to maintain copper homeostasis when encountering copper stress. Research on copper metabolism mechanisms in yeast was relatively clearer [2325], which included the uptake, storage, mitigation, and secretion of copper ions. Using yeast metabolism as reference, we analyzed genes associated with copper metabolism in wild-type T. harzianum Th33 and the mutant $\Delta$ thmeal under copper stress. In yeast, copper ions were reduced by the copper and iron reductase Frelp/Fre2p on the plasma membrane into $\mathrm{Cu}^{+}$ions before uptake. Subsequently, these ions were captured by the high-affinity copper transporter protein Ctrlp/Ctr3p or the low-affinity copper transporter protein Ctr2p and transported into cells $[26,27]$. The present study determined that, under copper stress, the copper reductase gene Tha_07043 (Fre2) was significantly upregulated in the $\Delta$ thmeal mutant, but both strains showed relatively low expression levels. This finding suggested that the intake of copper ions in both strains was relatively low. Similarly, the gene expression levels of four copper transporter proteins (Ctrp), namely, Tha_01417, Tha_02284, Tha_03591, and Tha_05828, in both strains were relatively low. This showed that thmeal may not have a strong correlation with intracellular transport of copper ions. However, whether there are other copper transportation channels present in Trichoderma requires further study. In yeast, $\mathrm{Cu}^{+}$ions that enter the cell were mainly bound by three copper chaperone proteins and transported to corresponding target molecules and organelles. The copper chaperone protein Ccslp transported coppers to copper and zinc superoxide dismutase Sodlp to remove intracellular reactive oxygen species and alleviate oxidative stress in cells $[28,29]$. Coxl7 was responsible for transporting copper ions to the mitochondria and SCO2 mediated the transport of copper ions into the cytochrome c oxidase pathway [30]. Atxlp transported bound copper ions to the Golgi bodies, which then transported $\mathrm{Cu}^{+}$to the Golgi vesicular system by the P-type ATPase Ccc2p that was located on the Golgi bodies [31, 32]. Finally, these ions were utilized in the synthesis of the multicopper oxidase Fet $3 p$ and secreted along with this protein [33]. This process played a crucial role in regulating intracellular copper homeostasis [34]. Our study found that the copper chaperone genes Tha_01680 (Ccs1) and Tha_07893 (Cox17) were not differentially expressed in the wild-type and $\Delta$ thmeal strains under copper stress, but both of them were expressed at low transcriptional level, whereas the copper and zinc superoxide dismutase gene Tha_04932 (Sod1) was upregulated in both strains, with the wild-type Th33 strain showing higher levels than the mutant strain. Therefore, we hypothesized that the

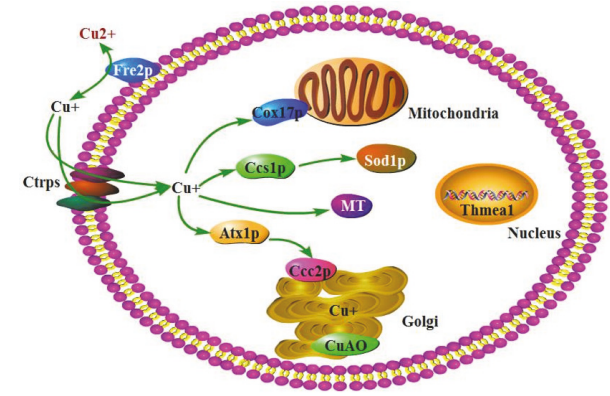

FIgURe 6: Proposed copper metabolism diagram in Trichoderma harzianum.

T. harzianum copper chaperone proteins Ccslp and Cox17p were not regulated by thmeal. However, Sodlp could clear reactive oxygen species in cells, thereby suggesting that thmeal may have some role in the regulation of superoxide dismutase expression. The copper chaperone gene Tha_00261 (Atxl) and the P-type ATPase gene Tha_08191 (Ccc2) showed upregulated expression in the mutant $\Delta$ thmeal. It was possible that the uptake rate of copper ions into the Golgi vesicular secretion system of the mutant strain $\Delta$ thmeal was relatively higher, and there were no changes in the transcript levels of the multicopper oxidase gene Tha_02340 (Fet3), which was responsible for the secretion of copper ions. However, the expression levels of the copper amine oxidase gene Tha_03788 $(\mathrm{CuAO})$ increased in the knockout mutant $\Delta$ thmeal. We speculated that knocking out the thmeal gene increased the ability of T. harzianum to secrete intracellular copper ions and Tha_03788 might (CuAO) have a similar function to the multicopper oxidase gene Fet 3 in T. harzianum and required further validation. Based on the above results and the findings of De Freitas et al. [23] on yeast copper metabolism, we described the copper metabolic diagram of T. harzianum (Figure 6). We hypothesized that after T. harzianum lost its thmeal gene, the ability of cells to scavenge reactive oxygen species, mainly through the glutathione antioxidant system, was enhanced, whereas protein synthesis and repair and copper secretion increased under copper stress, which increased the ability of the mutant strain to tolerate copper stress.

\section{Conclusions}

The $\mathrm{C} 2 \mathrm{H} 2$ transcription factor gene thmeal is a negative regulated factor of copper tolerance ability in T. harzianum and does not show metallothionein expression activator activities. Lacking of thmeal increases the copper tolerance of T. harzianum, and the ability of $\Delta$ thmeal to scavenge reactive oxygen species, mainly through the glutathione antioxidant system, is enhanced, protein synthesis and repair ability are enhanced by upregulating the expression of hot shock proteins and ribosomal proteins, and the secretion ability of copper irons is enhanced by upregulating the expression of copper chaperone protein Tha_00261 (Atxl), the P-type ATPase Tha_08191 (Ccc2), and copper amine oxidase gene Tha_03788 (CuAO), which increases the ability of the mutant strain to tolerate copper stress. 


\section{Data Availability}

The data used to support the findings of this study are included within the article.

\section{Conflicts of Interest}

The authors declare that there are no conflicts of interest regarding the publication of this paper.

\section{Acknowledgments}

The authors are grateful for the financial support from the National Natural Science Foundation of China (Grant no. 31371983), which funded the research and publication of this article.

\section{Supplementary Materials}

The supplementary material contains 7 tables and 1 figure. Table S1: primers used in validation experiments. Table S2: mapping rate of sequencing samples of Th33 and $\Delta$ thmeal. Table S3: gene number of different expression level (FPKM) distribution. Table S4: list of differentially expressed genes (DEGs) showed in Figure 2. Table S5: number of DEGs assigned to GO classification. Table S6: summary of DEGs assigned to the KEGG database. Table S7: list of 65 ribosomal protein genes with significantly altered expression in $T$. harzianum. Figure S1: the melting curve of the tested genes analyzed by qRT-PCR. (Supplementary Materials)

\section{References}

[1] R. Phieler, A. Voit, and E. Kothe, "Microbially supported phytoremediation of heavy metal contaminated soils: strategies and applications," Advances in Biochemical Engineering/Biotechnology, vol. 141, pp. 211-235, 2014.

[2] A. G. Babu, J. Shim, K.-S. Bang, P. J. Shea, and B.-T. Oh, "Trichoderma virens PDR-28: A heavy metal-tolerant and plant growth-promoting fungus for remediation and bioenergy crop production on mine tailing soil," Journal of Environmental Management, vol. 132, pp. 129-134, 2014.

[3] O. G. Oladipo, O. O. Awotoye, A. Olayinka, C. C. Bezuidenhout, and M. S. Maboeta, "Heavy metal tolerance traits of filamentous fungi isolated from gold and gemstone mining sites," Brazilian Journal of Microbiology, vol. 49, no. 1, pp. 29-37, 2018.

[4] L.-G. Li, L. Cai, X.-X. Zhang, and T. Zhang, "Potentially novel copper resistance genes in copper-enriched activated sludge revealed by metagenomic analysis," Applied Microbiology and Biotechnology, vol. 98, no. 24, pp. 10255-10266, 2014.

[5] J. Li, Y.-M. Zheng, Y.-R. Liu, Y.-B. Ma, H.-W. Hu, and J. Z. He, "Initial copper stress strengthens the resistance of soil microorganisms to a subsequent copper stress," Microbial Ecology, vol. 67, no. 4, pp. 931-941, 2014.

[6] M. A. Bouhlel, M. Lambert, and M.-H. David-Cordonnier, "Targeting transcription factor binding to dna by competing with dna binders as an approach for controlling gene expression," Current Topics in Medicinal Chemistry, vol. 15, no. 14, pp. 1323-1358, 2015.
[7] Q. Sun, X. Jiang, L. Pang, L. Wang, and M. Li, "Functions of thgal Gene in Trichoderma harzianum based on transcriptome analysis," BioMed Research International, vol. 2016, Article ID 8329513, pp. 1-9, 2016.

[8] M. Zabka and R. Pavela, "Antifungal efficacy of some natural phenolic compounds against significant pathogenic and toxinogenic filamentous fungi," Chemosphere, vol. 93, no. 6, pp. 10511056, 2013.

[9] C. Trapnell, B. A. Williams, G. Pertea et al., "Transcript assembly and quantification by RNA-Seq reveals unannotated transcripts and isoform switching during cell differentiation," Nature Biotechnology, vol. 28, no. 5, pp. 511-515, 2010.

[10] A. Conesa and S. Götz, "Blast2GO: a comprehensive suite for functional analysis in plant genomics," International Journal of Plant Genomics, vol. 2008, Article ID 619832, pp. 1-12, 2008.

[11] Y. Chai, S. L. Koppenhafer, N. M. Bonini, and H. L. Paulson, "Analysis of the role of heat shock protein (Hsp) molecular chaperones in polyglutamine disease," The Journal of Neuroscience, vol. 19, no. 23, pp. 10338-10347, 1999.

[12] K. Fu, L. Liu, L. Fan, T. Liu, and J. Chen, "Accumulation of copper in Trichoderma reesei transformants, constructed with the modified Agrobacterium tumefaciens-mediated transformation technique," Biotechnology Letters, vol. 32, no. 12, pp. 1815-1820, 2010.

[13] M. Ma, M. J. Christensen, and Z. Nan, "Effects of the endophyte Epichloëfestucae var. lolii of perennial ryegrass (Lolium perenne) on indicators of oxidative stress from pathogenic fungi during seed germination and seedling growth," European Journal of Plant Pathology, vol. 141, no. 3, pp. 571-583, 2015.

[14] A. Ahmed, A. Malik, H. Jagirdar et al., "Copper-induced inactivation of camel liver glutathione S-transferase," Biological Trace Element Research, vol. 169, no. 1, pp. 69-76, 2016.

[15] J. H. Freedman, M. R. Ciriolo, and J. Peisach, "The role of glutathione in copper metabolism and toxicity," The Journal of Biological Chemistry, vol. 264, no. 10, pp. 5598-5605, 1989.

[16] A. Corazza, I. Harvey, and P. J. Sadler, "1H,13C-NMR and Xray absorption studies of copper(I) glutathione complexes," European Journal of Biochemistry, vol. 236, no. 2, pp. 697-705, 1996.

[17] J. R. Warner and K. B. McIntosh, "How common are extraribosomal functions of ribosomal proteins?" Molecular Cell, vol. 34, no. 1, pp. 3-11, 2009.

[18] W. Vidal Magalhaes, M. F. Gouveia Nogueira, and T. M. Kaneko, "Heat Shock Proteins (HSP): dermatological implications and perspectives," European Journal of Dermatology, vol. 22, no. 1, pp. 8-13, 2012.

[19] G. Butler and D. J. Thiele, "ACE2, an activator of yeast metallothionein expression which is homologous to SWI5," Molecular and Cellular Biology, vol. 11, no. 1, pp. 476-485, 1991.

[20] P. R. Dohrmann, G. Butler, K. Tamai et al., "Parallel pathways of gene regulation: Homologous regulators SWI5 and ACE2 differentially control transcription of $\mathrm{HO}$ and chitinase," Genes \& Development, vol. 6, no. 1, pp. 93-104, 1992.

[21] J. N. Hegelund, M. Schiller, T. Kichey et al., "Barley metallothioneins: MT3 and MT4 are localized in the grain aleurone layer and show differential zinc binding," Plant Physiology, vol. 159, no. 3, pp. 1125-1137, 2012.

[22] L. T. Jensen, W. R. Howard, J. J. Strain, D. R. Winge, and V. C. Culotta, "Enhanced effectiveness of copper ion buffering by CUP1 metallothionein compared with CRS5 metallothionein in Saccharomyces cerevisiae," The Journal of Biological Chemistry, vol. 271, no. 31, pp. 18514-18519, 1996. 
[23] J. De Freitas, H. Wintz, J. H. Kim, H. Poynton, T. Fox, and C. Vulpe, "Yeast, a model organism for iron and copper metabolism studies," BioMetals, vol. 16, no. 1, pp. 185-197, 2003.

[24] J. Xu, G. Chen, X. Sun, X. Fan, and L. You-Zhi, "Paths and determinants for Penicillium janthinellum to resist low and high copper," Scientific Reports, vol. 5, no. 1, 2015.

[25] J. S. Valentine and E. B. Gralla, "Delivering copper inside yeast and human cells," Science, vol. 278, no. 5339, pp. 817-818, 1997.

[26] D. J. Eide, "The molecular biology of metal ion transport in Saccharomyces cerevisiae," Annual Review of Nutrition, vol. 18, pp. 441-469, 1998.

[27] M. M. O. Peña, J. Lee, and D. J. Thiele, "A delicate balance: Homeostatic control of copper uptake and distribution," Journal of Nutrition, vol. 129, no. 7, pp. 1251-1260, 1999.

[28] P. Huppke, C. Brendel, G. C. Korenke et al., "Molecular and biochemical characterization of a unique mutation in CCS, the human copper chaperone to superoxide dismutase," Human Mutation, vol. 33, no. 8, pp. 1207-1215, 2012.

[29] M. Fukuoka, E. Tokuda, K. Nakagome, Z. Wu, I. Nagano, and Y. Furukawa, "An essential role of N-terminal domain of copper chaperone in the enzymatic activation of $\mathrm{Cu} / \mathrm{Zn}$-superoxide dismutase," Journal of Inorganic Biochemistry, vol. 175, pp. 208216, 2017.

[30] L. Banci, I. Bertini, S. Ciofi-Baffoni et al., "A structuraldynamical characterization of human Cox17," The Journal of Biological Chemistry, vol. 283, no. 12, pp. 7912-7920, 2008.

[31] A. Cankorur-Cetinkaya, S. Eraslan, and B. Kirdar, "Transcriptomic response of yeast cells to ATX1 deletion under different copper levels," BMC Genomics, vol. 17, no. 1, article no. 489, 2016.

[32] S. E. Abdel-Ghany, J. L. Burkhead, K. A. Gogolin et al., "AtCCS is a functional homolog of the yeast copper chaperone Ccs1/Lys7," FEBS Letters, vol. 579, no. 11, pp. 2307-2312, 2005.

[33] E. Sedlák, G. Žoldák, and P. Wittung-Stafshede, "Synergistic effects of copper sites on apparent stability of multicopper oxidase, Fet3p," International Journal of Molecular Sciences, vol. 19, no. 1, p. 269, 2018.

[34] R. Safaei, M. H. Maktabi, B. G. Blair, C. A. Larson, and S. B. Howell, "Effects of the loss of Atoxl on the cellular pharmacology of cisplatin," Journal of Inorganic Biochemistry, vol. 103, no. 3, pp. 333-341, 2009. 


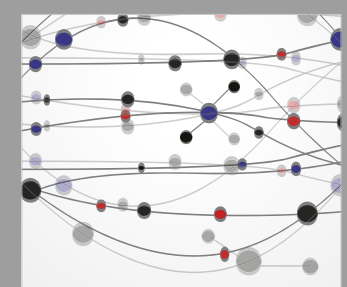

The Scientific World Journal
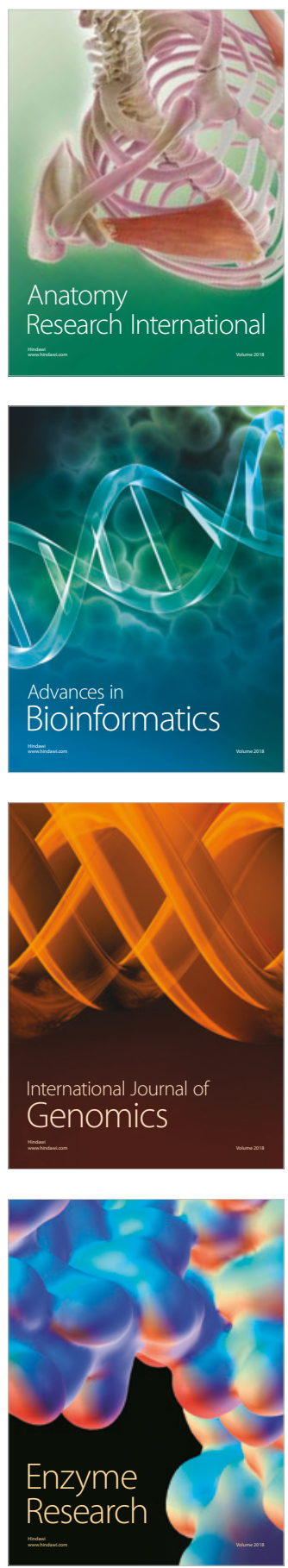
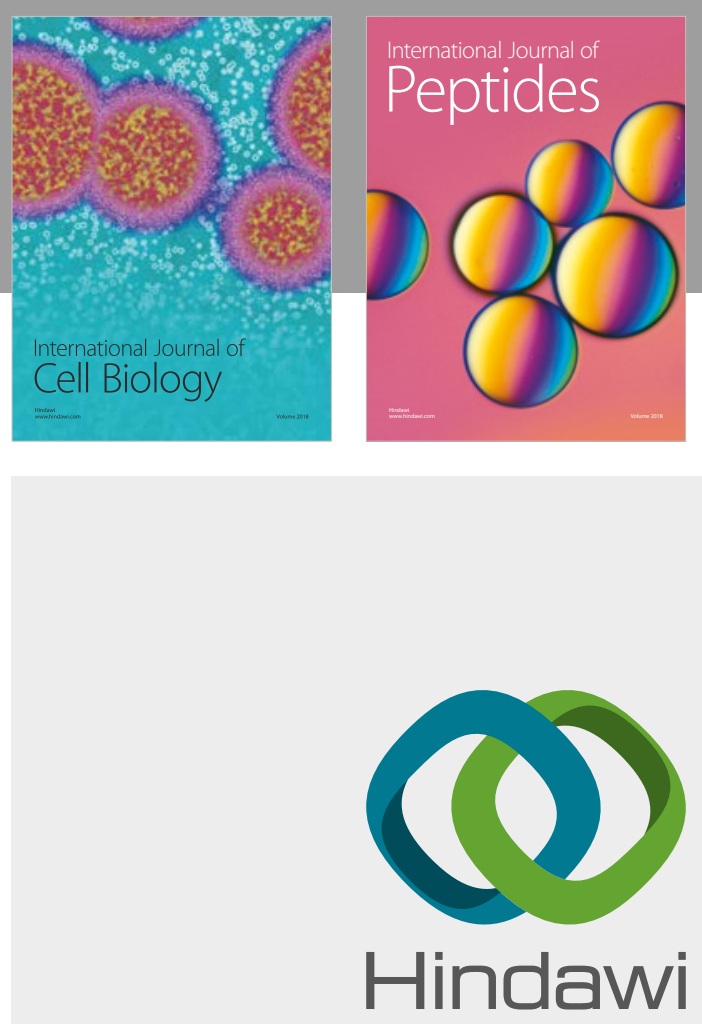

Submit your manuscripts at

www.hindawi.com
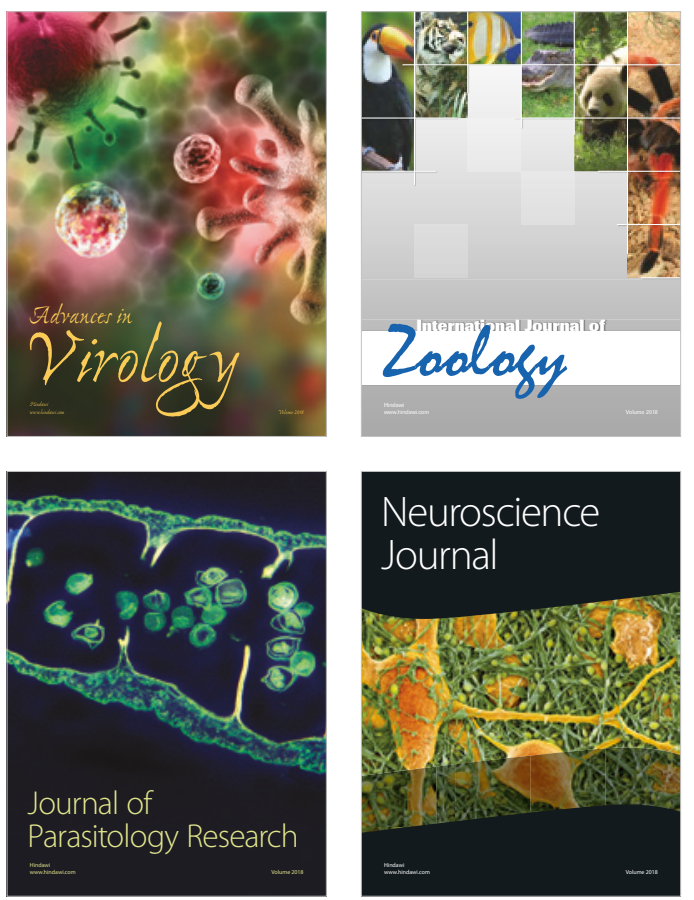
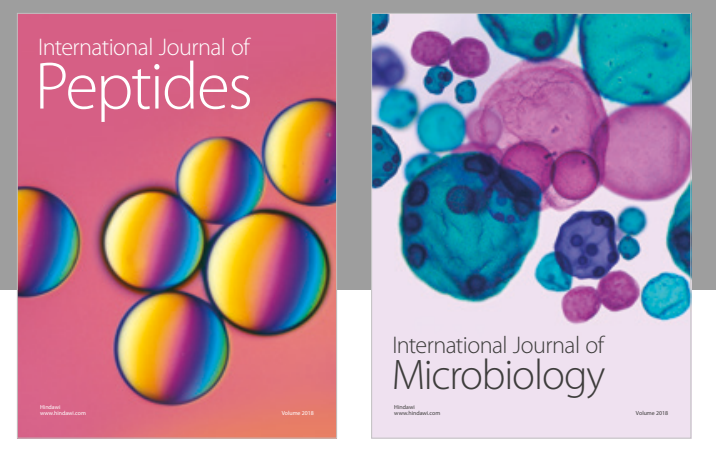

nternational Journal of Microbiology
Journal of
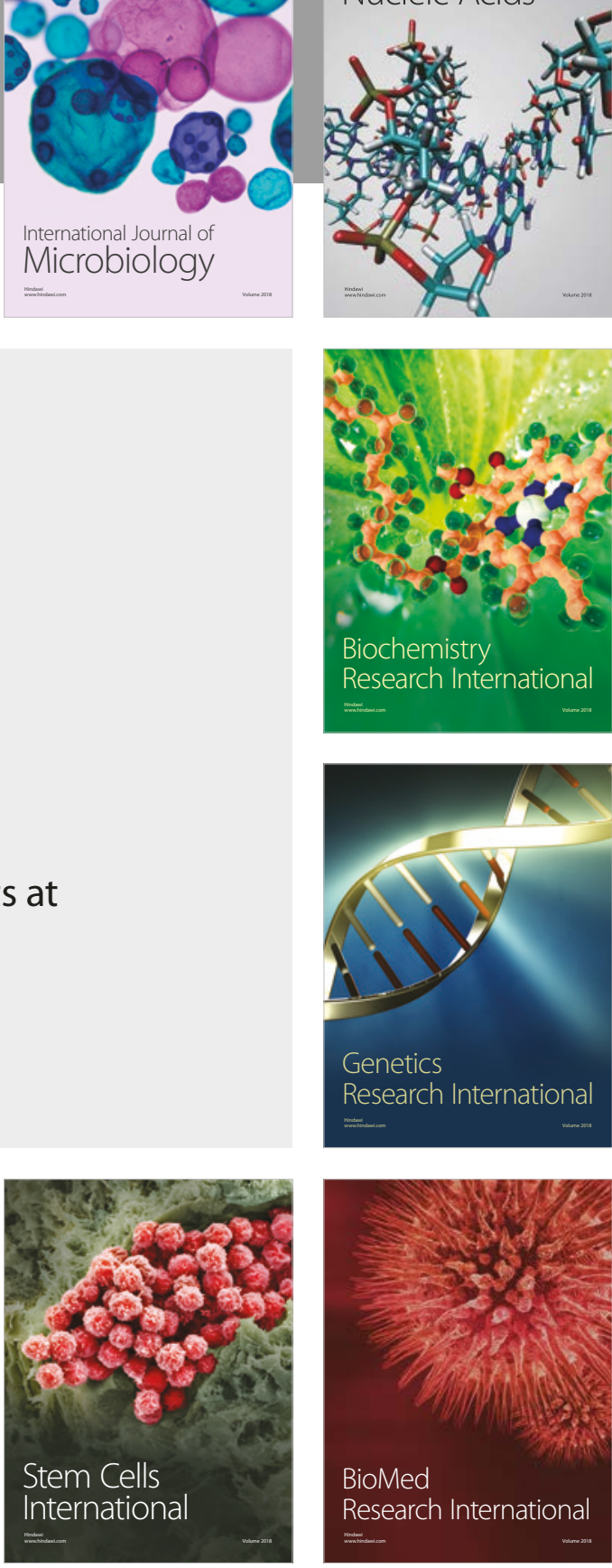
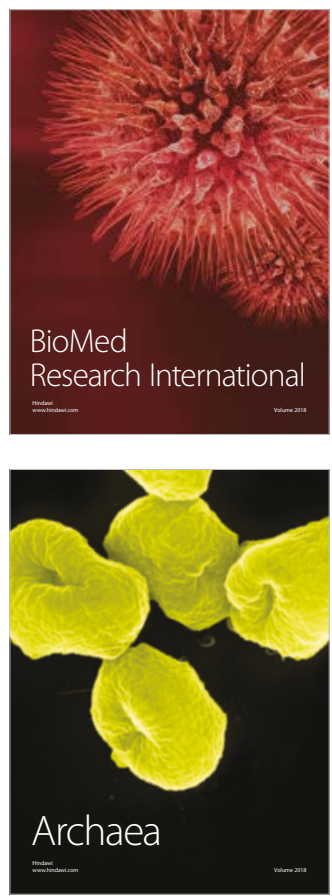